\title{
Multimodal Chemical and Physical Surface Characterization on a Combined AFM-MS Platform.
}

\author{
Olga S. Ovchinnikova ${ }^{1}$, Gary J. Van Berkel ${ }^{1}$ \\ ${ }^{1}$ Organic and Biological Mass Spectrometry Group, Chemical Sciences Division, Oak Ridge National \\ laboratory, Oak Ridge, TN 37831-6131, USA.
}

The need for better analytical tools that can provide high sensitivity, detailed molecular information with high spatial resolution as well as coregisted physical and temporal information is well recognized and is evidenced by the fact that it is a goal sought by many researchers and the impetus behind the development of several multimodal imaging techniques. Combining atomic force microscopy (AFM) and mass spectrometry (MS) onto one platform achieving molecular identification with correlated physical information has been demonstrated by our group as a method for high resolution spot sampling [1] and imaging [2] of test substrates. To continue to improve the performance metrics of our basic approach and to expand the ability to visualize the spatial distribution of a wider range of molecular species, we have worked to better understand and advance the material sampling, transfer and ionization components of the experiment. In this presentation, we will discuss a new inline atmospheric pressure sampling/APCI interface and the use of AFM probes with a higher maximum temperature as well as the broader range of compounds analyzed and surfaces imaged.

A comparison of the new AFM-MS coupling interface with the prior system we used on this mass spectrometer showed a signal increase of about a factor of 9 for the model system pressed pellet of rubrene. This was attributed to the shorter transfer capillary length and to the in-line APCI source that provided a confined reaction region, possibly improving ionization efficiency and also improving transmission of ions into the mass spectrometer. Using this interface a variety of analytes were investigated for the ability to be thermal desorbed, ionized and mass analyzed with this system. Desorption spot sizes from pressed pellets of the pure materials were $1 \mu \mathrm{m}$ or less. New analytes successfully analyzed to date include the slip agent erucamide, nickel and vanadyl alkylporphyrins, nickel and copper tetraphenylporphyrins, copper phthalocyanine, the polycyclic aromatic hydrocarbons perlyene, chrysene, 8,10-diphenylanthracene and rubrene, as well as $\mathrm{C}_{60}$. Under optimized conditions, $\mathrm{C}_{60}$ could be detected from a desorption spot in a pressed pellet of the material desorption spot size of $750 \mathrm{~nm}$ wide and $150 \mathrm{~nm}$ deep. We also found that addition of a dopant into the interface "T" (e.g., toluene) could be used to alter the ionization chemistry of the source to promote formation of radical cations over protonated molecules. Using this interface multimodal co-registered imaging experiments were carried out on mouse brain tissue. Co-registered corresponding topographic and chemical images of endogenous compounds where acquired with $2.5 \mu \mathrm{m}$ x $2 \mu \mathrm{m}$ (x-axis by y-axis) chemical spatial resolution, and $0.2 \mu \mathrm{m} \times 0.78 \mu \mathrm{m}$ (x-axis by y-axis) topographic resolution. [3]

\section{References:}

[1] OS Ovchinnikova, MP Nikiforov, JA Bradshaw, S Jesse, GJ Van Berkel, ACS Nano 5 (2011), 5526-5531.

[2] OS Ovchinnikova, K Kjoller, GB Hurst, DA Pelletier, GJ Van Berkel, Anal. Chem. 86 (2014), 1083-1090. 
[3] Anasys Instruments for providing the loan AFM system. This work was supported by the Division of Chemical Sciences, Geosciences, and Biosciences, Office of Basic Energy Sciences, United States Department of Energy. ORNL is managed by UT-Battelle, LLC for the U.S. Department of Energy under contract DE-AC05-00OR22725.

Figure 1. Multimodal imaging. (a) Optical image of yellow ink stamped onto photo paper. (d) Corresponding AFM surface topography image overlaid with the interpolated mass spectral chemical image using the extracted ion current from major product ion of Pigment Yellow 74 in yellow ink $\mathrm{m} / \mathrm{z}$ 264. The imaging data was acquired using MS/MS (m/z $387 \rightarrow \mathrm{m} / \mathrm{z} 264$, normalized collision energy $25 \%$ ) in positive ion mode APCI with a fixed trap fill time of $100 \mathrm{~ms}$, surface scan rate of $10 \mu \mathrm{m} / \mathrm{s}$, lane spacing of $2 \mu \eta$ and AFM probe tip temperature of $350^{\circ} \mathrm{C}$.

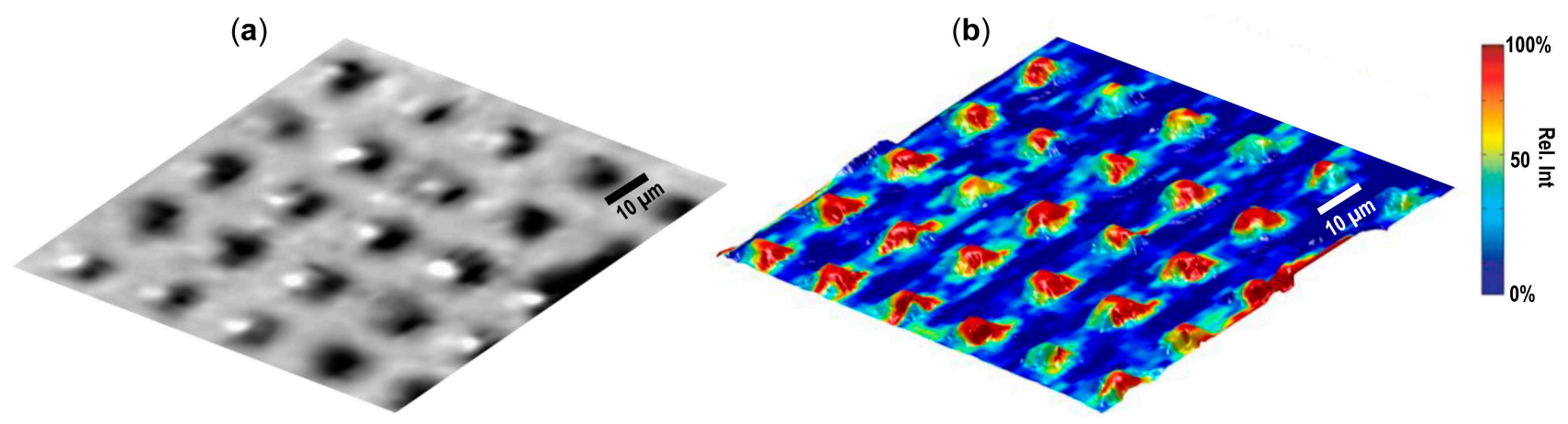

